\title{
The Glutamate-Activated Anion Conductance in Excitatory Amino Acid Transporters Is Gated Independently by the Individual Subunits
}

\author{
Hans Peter Koch, Ronald Lane Brown, and Hans Peter Larsson \\ Neurological Sciences Institute, Oregon Health \& Science University, Beaverton, Oregon 97006
}

\begin{abstract}
Excitatory amino acid transporters (EAATs) use sodium and potassium gradients to remove glutamate from the synapse and surrounding extracellular space, thereby sustaining efficient synaptic transmission and maintaining extracellular glutamate concentrations at subneurotoxic levels. In addition to sodium-driven glutamate uptake, EAATs also mediate a glutamate-activated chloride conductance via a channel-like mechanism. EAATs are trimeric proteins and are thought to comprise three identical subunits. Previous studies have shown that the sodium-driven uptake of glutamate occurs independently in each of the three subunits. In contrast, a recent study reports high Hill coefficients for the activation of EAAT anion currents by glutamate and suggests that the subunits function cooperatively in gating the chloride conductance. In the present work, we find that the Hill coefficient for the activation of the anion current by glutamate is $\sim 1$ in both EAAT3 and EAAT4. Furthermore, we also used fluorescent labeling and inactivation correlation on EAAT3 and EAAT4 to determine whether the glutamate-activated chloride conductance is gated independently or cooperatively by the transporters. We found that both glutamate uptake currents and glutamate-activated chloride currents are mediated independently by each subunit of an EAAT multimer. It has been suggested that EAAT subtypes with particularly large anion conductances can directly influence the excitability of presynaptic terminals in certain neurons. Thus, the finding that the anion conductance is gated independently, rather than cooperatively, is important because it significantly alters predictions of the influence that EAAT-mediated anion currents will have on synaptic transmission at low glutamate concentrations.
\end{abstract}

Key words: anion conductance; chloride; independence; cooperativity; FLIC; glutamate transporter

\section{Introduction}

Glutamate is the predominant excitatory neurotransmitter in the mammalian CNS, and excitatory amino acid transporters (EAATs) facilitate its reuptake into neurons and glia. EAATs are consequently responsible for modulating excitatory glutamatergic signaling, recycling the neurotransmitter, and preventing neuronal damage attributable to high levels of extracellular glutamate. Five subtypes of EAATs have been cloned (EAAT1EAAT5), and they are expressed ubiquitously in neurons and glia (for review, see Danbolt, 2001). EAATs transport glutamate against its concentration gradient by coupling uptake to sodium and potassium gradients. Uptake of one glutamate molecule is accompanied by the cotransport of three $\mathrm{Na}^{+}$and one $\mathrm{H}^{+}$and the counter-transport of one $\mathrm{K}^{+}$, resulting in a net inward coupled charge movement of +2 (Zerangue and Kavanaugh, 1996). In addition to the coupled uptake current, EAATs also mediate an anion (chloride) conductance that exhibits channel-like properties (Fairman et al., 1995; Picaud et al., 1995b; Wadiche et al.,

Received 0ct. 2, 2006; revised Feb. 12, 2007; accepted Feb. 15, 2007.

This study was supported by National Institutes of Health Grants T32-NS045553 (H.P.K.), MH67094 (R.L.B.), and NS051169 (H.P.L.).

Correspondence should be addressed to Hans Peter Larsson, Neurological Sciences Institute, Oregon Health \& Science University, Beaverton, OR 97006. E-mail: larssonp@ohsu.edu.

D01:10.1523/JNEUROSCI.0118-07.2007

Copyright $\odot 2007$ Society for Neuroscience $\quad$ 0270-6474/07/272943-05\$15.00/0 1995b; Larsson et al., 1996; Arriza et al., 1997). Although it is activated by glutamate, this anion conductance is thermodynamically uncoupled from glutamate uptake, and thus glutamate uptake is unaffected by anion concentrations (Wadiche et al., 1995a). Although the role of the anion conductance in EAATs is unclear, it has been postulated that EAAT subtypes with particularly high anion permeability may contribute directly to excitability in certain cells (Picaud et al., 1995a; Palmer et al., 2003; Veruki et al., 2006; Wersinger et al., 2006).

EAATs are multimeric proteins composed of three identical subunits (Yernool et al., 2004; Koch and Larsson, 2005), and crystallographic structures reveal an individual glutamate binding site in each of the subunits (Yernool et al., 2004). Previous work has demonstrated that each subunit of an EAAT transports glutamate independently (Grewer et al., 2005; Koch and Larsson, 2005). In contrast, a recent study suggested that the individual subunits of EAAT4 function cooperatively in gating the anion conductance. The authors reported a sigmoidal concentration dependence for glutamate with Hill coefficients $\geq 3$ and suggested that multiple glutamate binding sites must be occupied to activate the anion conductance (Torres-Salazar and Fahlke, 2006).

In the present work, we examine whether the anion conductance is activated independently or cooperatively by the individual subunits of EAATs. We measured the concentration depen- 
dence by which glutamate activates the anion conductance in the neuronal transporters EAAT3 and EAAT4, EAAT4 being a subtype with one of the highest anion permeabilities among glutamate transporters. In addition, we used fluorescence labeling and inactivation correlation (FLIC) on EAAT3 and EAAT4 to simultaneously label and inactivate individual subunits. We found that the anion conductance is activated independently by each individual subunit of an EAAT multimer under all conditions studied.

\section{Materials and Methods}

Expression of EAATs in oocytes and oocyte electrophysiology. Mutagenesis, in vitro RNA synthesis, and RNA injection into Xenopus laevis oocytes were performed as described previously (Larsson et al., 2004). Currents were measured in two-electrode voltage-clamp configuration as described previously (Larsson et al., 2004). Glutamate-activated currents were calculated by subtracting current measured in $\mathrm{NaCl}-, \mathrm{NaSCN}-$, or $\mathrm{Na}$-gluconate-based Ringer's solution from current measured in Ringer's solution containing various concentrations of glutamate.

Fluorescence measurements. Individual oocytes were voltage clamped and labeled with tetramethylrhodamine-methanethiosulfonate (TMRMTS) (Toronto Research Chemicals, North York, Ontario, Canada) in $\mathrm{NaCl}-$ or NaSCN-Ringer's solution. Exposure time and fluorophore concentration were varied for the indicated time points (e.g., in Fig. $2 \mathrm{~A}$, each oocyte was exposed to $25 \mu \mathrm{M}$ TMR-MTS for $30 \mathrm{~s}$ for the first time point, an additional $30 \mathrm{~s}$ for the second time point, etc). Fluorescence was measured using an upright microscope (Leica, Nussloch, Germany) equipped with a $20 \times$ quartz objective, rhodamine filter cube (exciter, HQ545/30×; dichroic, Q570LP; emitter, HQ620/60m), and photodiode (pin-020A; UDT Sensors, Hawthorne, CA) coupled to an Axopatch 200B patch-clamp amplifier (Molecular Devices, Palo Alto, CA). The objective was focused on the animal pole, and fluorescence was monitored with Clampex 8.2 (Molecular Devices). Oocytes were thoroughly washed in Ringer's solution between each fluorescence measurement to remove any unbound fluorophore, and glutamate-induced currents were subsequently measured at each time point. For subtraction of background fluorescent labeling, uninjected oocytes were exposed to fluorescent label under identical conditions, and transporter-specific fluorescent labeling was obtained by subtracting this background fluorescence at each time point.

In the FLIC experiments, the three subunits are assumed to be labeled independently by the fluorescent probe. The probability that a subunit will be labeled is $p[t]=1-\exp (-t / \tau)$, where $\tau$ is the measured labeling time constant and $\mathrm{t}$ is exposure time. The probability that no subunit is labeled is $p_{0-\text { of- } 3 \text { subunits }}[t]=(1-p[t])^{3}=\exp (-3 t / \tau)$. The probability that any subunit in the trimer is labeled is then $p_{\text {any-of- } 3 \text { subunits }}[t]=1-$ $p_{0 \text {-of-3 subunits }}[t]=1-\exp (-3 t / \tau)$. If the current is activated independently by each subunit, then the current will decrease by the same time course as $p[t]=1-\exp (-t / \tau)$. If the current is activated only if all three subunits have bound glutamate, i.e., if the labeling of one subunit is sufficient to block current in the entire multimer, then the current will decrease by the same time course as $p_{\text {any-of-3 subunits }}[t]=1-\exp (-3 t / \tau)$.

Culture, transfection, and whole-cell recordings of HEK-293 cells. Human embryonic kidney HEK-293 cells were maintained and transfected as described previously (Brady et al., 2006). Three to $5 \mathrm{~d}$ after transfection, cells were lifted by trituration and placed in a recording chamber mounted on an inverted microscope (Nikon, Tokyo, Japan). Internal solution contained the following (in mM): $115 \mathrm{NaCl}, 2 \mathrm{MgCl}_{2}, 5 \mathrm{EGTA}$, and 10 HEPES, $\mathrm{pH}$ 7.4. External solution contained the following (in $\mathrm{mm}$ ): $140 \mathrm{NaSCN}, 4 \mathrm{KCl}, 2 \mathrm{CaCl}_{2}, 1 \mathrm{MgCl}_{2}$, and 5 HEPES, pH 7.4, supplemented with glutamate at indicated concentrations. Transfected cells containing enhanced green fluorescent protein (EGFP) were identified by epifluorescence microscopy. Pipette resistances were 3-5 M $\Omega$, and currents were recorded in whole-cell mode using an Axopatch 200A amplifier (Molecular Devices); voltage commands and data acquisition were driven by pClamp 8.0 software (Molecular Devices). After obtaining the whole-cell configuration, cell capacitance and series resistance were measured and compensated by $70 \%$. Currents were filtered at $2 \mathrm{kHz}$
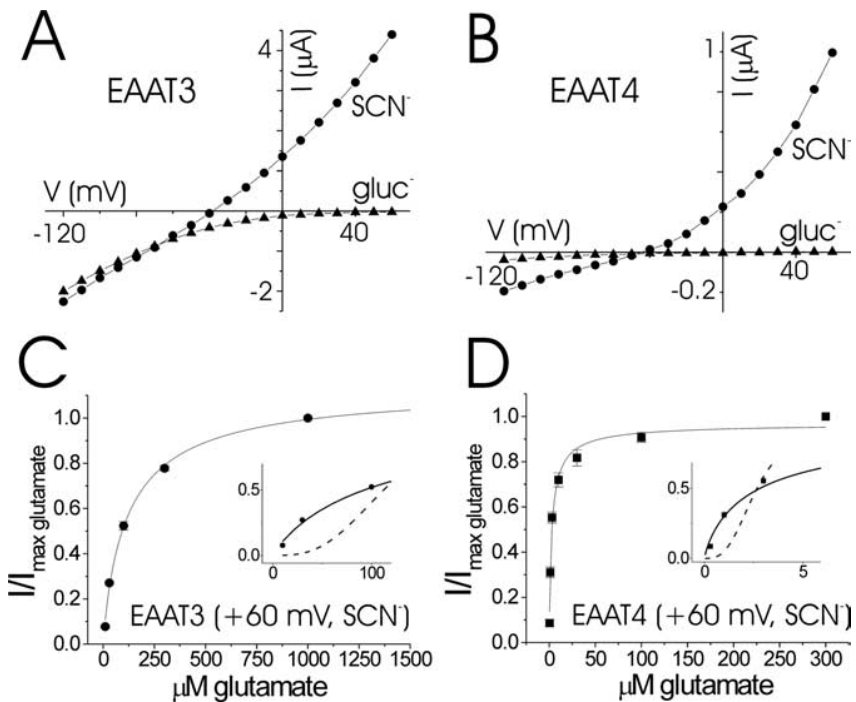

Figure 1. Glutamate-induced currents in EAAT3 and EAAT4. Representative current-voltage relationships for $1 \mathrm{~mm}$ glutamate application to oocytes expressing EAAT3 $(\boldsymbol{A})$ and EAAT4 $(\boldsymbol{B})$. - , Current recorded in $\mathrm{NaSCN}-$ Ringer's solution $\left(\mathrm{SCN}^{-}\right)$; $\mathbf{\Delta}$, current from cells dialyzed in gluconate-Ringer's solution for $24 \mathrm{~h}$ and recorded in gluconate-Ringer's solution (gluc ${ }^{-}$). $C_{\text {, }}$ Dose-response for glutamate currents in EAAT3 recorded in NaSCN-Ringer's solution at +60 $\mathrm{mV} ; K_{\mathrm{m}}=122.2 \pm 15 \mu \mathrm{M}, n_{\mathrm{H}}=0.91 \pm 0.10(n=3)$. D, Dose-response for glutamate currents in EAAT4 recorded in NaSCN-Ringer's solution at $+60 \mathrm{mV} ; K_{\mathrm{m}}=2.62 \pm 0.55 \mu \mathrm{M}$, $n_{\mathrm{H}}=0.91 \pm 0.13(n=3)$. Insets in $\boldsymbol{C}, \boldsymbol{D}$, Expanded view of experimental data fit with the Hill equation (solid curves) versus simulated data fit with the Hill equation with identical $K_{\mathrm{m}}$ and $I / I_{\text {max glu }}$ as in experimental data but with $n_{\mathrm{H}}=3$ (dashed curves).

and sampled at $10 \mathrm{kHz}$. Cells were superfused with a series of glutamate concentrations using a rapid solution changer (RSC-100; Biologic; Molecular Kinetics, Pullman, WA). Glutamate-activated currents were determined by subtraction of currents measured in the absence of glutamate.

\section{Results}

In the present study, we measured the concentration dependence by which glutamate activates currents in EAAT3 and EAAT4. Consistent with previous results, the affinity of EAAT3 for glutamate at $-80 \mathrm{mV}$ in NaCl-Ringer's solution was $K_{\mathrm{m}}=38.1 \pm 6$ $\mu \mathrm{M}$ with $n_{\mathrm{H}}=1.1 \pm 0.04(n=5)$, and the affinity of EAAT4 for glutamate was $K_{\mathrm{m}}=0.90 \pm 0.16 \mu \mathrm{M}$ with $n_{\mathrm{H}}=1.09 \pm 0.21(n=$ 4) (data not shown). Under these physiological conditions, EAAT-mediated currents consist of two components: an inward current that is coupled to glutamate uptake, as well as an uncoupled chloride conductance. At positive potentials, however, EAATs generate outward currents that reflect only the uncoupled anion current (Wadiche et al., 1995b). To verify this, we measured glutamate-activated currents in EAAT3 and EAAT4 in the absence of chloride [in gluconate-Ringer's solution (in $\mathrm{mM}$ ): 98 Na-gluconate, 1.8 Ca-gluconate, $1 \mathrm{Mg}$-gluconate, and 5 HEPES, $\mathrm{pH} 7.5$ ] in oocytes that had been depleted of intracellular chloride by $24 \mathrm{~h}$ of dialysis in gluconate-Ringer's solution. In both EAAT3 and EAAT4, currents could not be detected at potentials above $+30 \mathrm{mV}$ in gluconate-Ringer's solution (Fig. $1 A, B$ ).

To further study the anion currents in isolation, we next recorded the glutamate-activated currents in EAAT3 and EAAT4 in NaSCN-Ringer's solution (in mM: $20 \mathrm{NaSCN}, 78 \mathrm{NaCl}, 1.8$ $\mathrm{CaCl}_{2}, 1 \mathrm{MgCl}_{2}$, and 5 HEPES, pH 7.5). Chaotropic ions such as $\mathrm{SCN}^{-}$have been shown to amplify anion currents in EAATs (Wadiche and Kavanaugh, 1998). Under these conditions, large outward anion currents were seen in both EAAT3 and EAAT4 at positive potentials in SCN-Ringer's solution (Fig. $1 A, B$ ). There- 
fore, at voltages more positive than $+30 \mathrm{mV}$, only the uncoupled anion conductance, but not the coupled glutamate conductance, is present in EAAT3 and EAAT4 (Fig. $1 A, B$ ). Furthermore, even at negative potentials, the majority of current in EAAT4 is carried by anions (Fig. 1B) (Fairman et al., 1995).

To completely isolate the anion current and to determine its dependence on glutamate concentration, we recorded glutamateinduced currents in oocytes expressing EAAT3 and EAAT4 at $+60 \mathrm{mV}$ in NaSCN-Ringer's solution. The glutamate affinity of both EAAT3 and EAAT4 was approximately threefold lower at $+60 \mathrm{mV}$ than at $-80 \mathrm{mV}$ (Fig. $1 C, D$ ), most likely reflecting the voltage dependence of $\mathrm{Na}^{+}$binding, which in turn affects glutamate affinity (Wadiche et al., 1995a; Larsson et al., 2004). In both EAAT3 and EAAT4, the concentration dependence of the glutamate-activated current recorded at $+60 \mathrm{mV}$ in $\mathrm{NaSCN}-$ Ringer's solution was well fit with the Hill equation, with $n_{\mathrm{H}}$ equal to $\sim 1$ (Fig. $1 C, D$ ), suggesting that the anion conductance is gated independently by the individual subunits in EAAT3 and EAAT4.

To further investigate whether individual subunits of the EAAT multimer interact cooperatively in gating the anion conductance, we used FLIC in oocytes expressing EAAT3 and EAAT4. We previously used FLIC in oocytes to demonstrate that glutamate transport occurs independently in each of the individual subunits of the EAAT3 multimer (Koch and Larsson, 2005). In EAAT3 transporters containing the point mutation S334C, glutamate-induced currents are inactivated when the introduced cysteine residue is covalently modified with the fluorescent probe TMR-MTS. This mutation allowed us to simultaneously monitor the fluorescence labeling of individual transporter subunits and the concurrent inactivation of transporter current. If the individual subunits of the transporter function independently, then the time course for the percentage decrease in current attributable to TMR-MTS labeling will correlate 1:1 with the time course for the increase in fluorescence attributable to TMR-MTS labeling. Conversely, if there is cooperativity between subunits, i.e., if all three subunits need to bind glutamate to activate the anion channel, then the time course for the decrease in current will be significantly faster, up to threefold, than the time course for the increase in fluorescence. In EAAT3-expressing oocytes, currents induced in $\mathrm{NaCl}$-Ringer's solution by bath application of $1 \mathrm{~mm}$ glutamate were monitored at -80 and $-30 \mathrm{mV}$. At $-80 \mathrm{mV}$, both the coupled glutamate-uptake current and the uncoupled glutamateactivated anion current are present, whereas at $-30 \mathrm{mV}$, which is the approximate chloride reversal potential, only the coupled glutamate-uptake current is detected. At both -80 and $-30 \mathrm{mV}$, the time course for the decrease in current attributable to TMRMTS labeling was nearly identical to the time course for the increase in fluorescence attributable to TMR-MTS labeling (Fig. $2 A$ ), demonstrating that the EAAT subunits function independently under these conditions. To isolate the anion conductance and to determine whether it also is independently gated by the individual subunits of the EAAT multimer, we measured FLIC in EAAT3 at $+60 \mathrm{mV}$ in NaSCN-Ringer's solution. Figure $2 \mathrm{~B}$ shows that the anion currents activated by $1 \mathrm{~mm}$ glutamate are decreased by TMR-MTS labeling with nearly the identical time course as the increase in fluorescence attributable to TMR-MTS labeling, demonstrating that the anion conductance is also activated independently by the individual EAAT subunits.

At saturating concentrations of glutamate, each subunit of the EAAT multimer will primarily be in either a glutamate-bound state or an intracellular-facing conformation. At lower glutamate concentrations, however, one or more subunits may have no
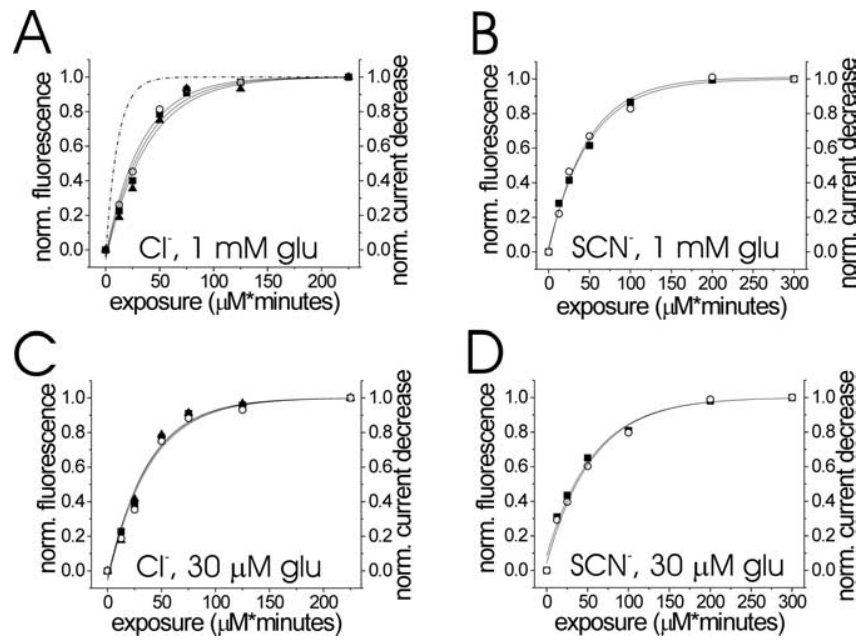

Figure 2. FLIC in EAAT3 S334C. $\boldsymbol{A}$, Fluorescence-labeling curve for EAAT3 S334C with TMRMTS in $\mathrm{NaCl}$-Ringer's solution (O) superimposed on the percentage decrease in current activated by $1 \mathrm{~mm}$ glutamate ( $\mathrm{glu}$ ) and recorded in $\mathrm{NaCl}-$ Ringer's solution at $-80 \mathrm{mV}$ ( $\square$ ) or -30 $\mathrm{mV}(\boldsymbol{\Delta})$. Dashed line indicates the predicted decrease in current for a concerted cooperative trimeric transporter (see Materials and Methods, Fluorescence measurements). For fluorescence labeling, $\tau=38.7 \pm 5.4 \mu \mathrm{m} \times \mathrm{min}$; for current decrease at $-80 \mathrm{mV}, \tau=36.1 \pm 3.8$ $\mu \mathrm{M} \times \mathrm{min}$; for current decrease at $-30 \mathrm{mV}, \tau=33.2 \pm 3.0 \mu \mathrm{m} \times \min$. $\boldsymbol{B}$, Fluorescencelabeling curve for EAAT3 S334C with TMR-MTS in NaSCN-Ringer's solution (O) superimposed on the percentage decrease in current activated by $1 \mathrm{~mm}$ glutamate and recorded in NaSCNRinger's solution at $+60 \mathrm{mV}(\square)$. For fluorescence labeling, $\tau=47.7 \pm 3.6 \mu \mathrm{m} \times \min$.; for current decrease at $+60 \mathrm{mV}, \tau=49.9 \pm 2.7 \mu \mathrm{m} \times \mathrm{min}$. C, Fluorescence-labeling curve for EAAT3 S334C with TMR-MTS in NaCl-Ringer's solution $(O)$ superimposed on the percentage decrease in current activated by $30 \mu \mathrm{m}$ glutamate and recorded in $\mathrm{NaCl}-$ Ringer's solution at $-80 \mathrm{mV}(\square)$ or $-30 \mathrm{mV}(\mathbf{\Delta})$. For fluorescence labeling, $\tau=38.6 \pm 5.5 \mu \mathrm{M} \times \mathrm{min}$; for current decrease at $-80 \mathrm{mV}, \tau=38.0 \pm 3.7 \mu \mathrm{m} \times$ min; for current decrease at $-30 \mathrm{mV}$, $\tau=36.9 \pm 3.9 \mu \mathrm{m} \times \min$. D, Fluorescence-labeling curve for EAAT3 S334C with TMR-MTS in NaSCN-Ringer's solution $(O)$ superimposed on the percentage decrease in current activated by $30 \mu \mathrm{m}$ glutamate and recorded in NaSCN-Ringer's solution at $+60 \mathrm{mV}(\square)$. For fluorescence labeling, $\tau=56.0 \pm 4.8 \mu \mathrm{m} \times$ min; for current decrease at $+60 \mathrm{mV}, \tau=56.3 \pm 6.2 \mu \mathrm{m} \times$ $\mathrm{min}$. In all experiments, the fluorescence from uninjected oocytes exposed to identical labeling conditions as in the experimental was subtracted as background. All data are representative for $n=3$ experiments.

glutamate bound and will thus be more influenced by the state of the neighboring subunits, provided there is indeed a cooperative interaction. In other words, the effect of intersubunit cooperativity should be larger at lower glutamate concentrations. We therefore measured FLIC in EAAT3 S334C at subsaturating concentrations of glutamate $(30 \mu \mathrm{M})$. We found that both the coupled transport current (recorded at -80 and $-30 \mathrm{mV}$ in $\mathrm{NaCl}-$ Ringer's solution) and the uncoupled anion conductance (recorded at $+60 \mathrm{mV}$ in NaSCN-Ringer's solution) were decreased by TMR-MTS labeling with similar time constants as the increase in fluorescence attributable to TMR-MTS labeling (Fig. $2 C, D)$, suggesting that the EAAT3 subunits function independently at all substrate concentrations. Although the fluorescent labeling time course was slightly faster at negative than at positive voltages (e.g., $\tau=38.6 \mu \mathrm{M} \times \min$ at $-80 \mathrm{mV}$ vs $\tau=56.3 \mu \mathrm{M} \times$ min at $+60 \mathrm{mV}$ ) (Fig. 2C,D), there may be several explanations for this. One possibility is that tetramethylrhodamine, which is protonated at physiological $\mathrm{pH}$, must traverse a small portion of the membrane electric field to access the introduced cysteine. Another possibility is that there is a small change in the accessibility of the cysteine itself when the voltage is changed from +60 to $-80 \mathrm{mV}$. Regardless, the difference in labeling time constant is relatively small ( $<1.5$-fold) and is inconsequential to the scope of the present work. 
EAAT4 has one of the highest chloride permeabilities among all the glutamate transporter subtypes (Fairman et al., 1995), and a recent study has suggested that the individual subunits of EAAT4 interact cooperatively in gating glutamate-activated anion currents (Torres-Salazar and Fahlke, 2006). We therefore measured FLIC in EAAT4 to determine whether or not its subunits interact cooperatively in gating the anion conductance. The analogous mutation to EAAT3 S334C in EAAT4 is S388C. Similar to EAAT3 S334C, transporter currents in EAAT4 S388C are abolished when the introduced cysteine is covalently modified with fluorophore. Using FLIC, we found that the anion current in EAAT4 S388C (activated by $300 \mu \mathrm{M}$ glutamate and recorded in $\mathrm{NaSCN}-$ Ringer's solution at $+60 \mathrm{mV}$ ) decreases in response to TMR-MTS labeling with a nearly identical time constant as the increase in fluorescence attributable to TMR-MTS labeling (Fig. 3). This suggests that the anion current in EAAT4 is also gated independently by its three individual subunits.

The most current kinetic models of EAATs suggest that, after glutamate translocation, the substrate binding sites can be reoriented to the extracellular face by binding either intracellular $\mathrm{K}^{+}$or $\mathrm{Na}^{+}$, depending on the composition of the intracellular solution (Bergles et al., 2002; Larsson et al., 2004). In our present work, as well as in most other studies examining glutamate currents in EAATs, physiological $\mathrm{K}^{+}$-based intracellular solutions were used. In contrast, in the recent work by Torres-Salazar and Fahlke, the authors eliminated internal $\mathrm{K}^{+}$and instead used $\mathrm{Na}^{+}$as the primary intracellular monovalent cation to study anion currents in EAAT4. Under these conditions, the authors reported a $K_{\mathrm{D}}$ for glutamate binding of $\geq 35 \mu \mathrm{M}$ and Hill coefficient of $\geq 3$ for glutamate activation of the anion currents in EAAT4 (Torres-Salazar and Fahlke, 2006). These findings are in sharp contrast to our present data as well as previous reports for glutamate-activated anion currents in EAAT4: $K_{\mathrm{D}}$ for glutamate binding $\approx 0.5-3 \mu \mathrm{M}$ with a Hill coefficient $\approx 1$ (Fairman et al., 1995; Mim et al., 2005). We initially hypothesized that it was the unusual recording conditions used in the study by Torres-Salazar and Fahlke that resulted in the higher than previously reported values for the $K_{\mathrm{D}}$ and Hill coefficient for glutamate binding. To test this hypothesis, we heterologously expressed rat EAAT4 in HEK-293 cells and measured glutamateactivated currents in the whole-cell patch-clamp configuration under conditions similar to those used by Torres-Salazar and Fahlke. Cells were dialyzed with $\mathrm{K}^{+}$-free solutions in which $\mathrm{Na}^{+}$ was the only intracellular monovalent cation, and a range of extracellular glutamate concentrations were applied in the presence of $\mathrm{SCN}^{-}$. These conditions induced the greatest degree of intersubunit cooperativity $\left(n_{\mathrm{H}}=5\right)$ in the work of Torres-Salazar and Fahlke (2006). In stark contrast to that work, however, we find that, under identical recording conditions, the $K_{\mathrm{m}}$ for glutamate is $1.018 \pm 0.188 \mu \mathrm{M}$ with the Hill coefficient $n_{\mathrm{H}}=0.997 \pm 0.085$ at $+80 \mathrm{mV}$ ( $n=4$ cells) (Fig. 4$)$. The values for $K_{\mathrm{m}}$ and $n_{\mathrm{H}}$ for glutamate currents did not change significantly across the range of positive voltages tested (Fig. 4A). This indicates that, regardless of whether $\mathrm{Na}^{+}$or $\mathrm{K}^{+}$is the monovalent cation available for intracellular binding, the individual EAAT subunits function independently during glutamate activation of anion currents.

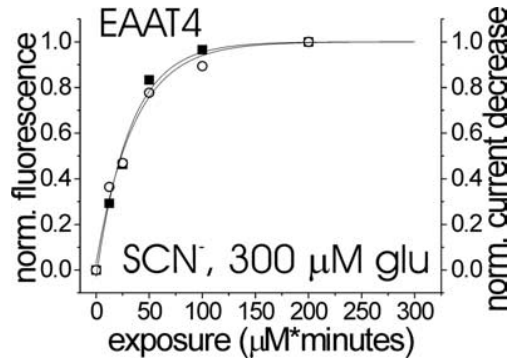

Figure 3. FLIC in EAAT4 S388C. Fluorescence-labeling curve for EAAT4 S388C with TMR-MTS in NaSCN-Ringer's solution $(O)$ superimposed on the percentage decrease in current activated by $300 \mu \mathrm{m}$ glutamate and recorded in NaSCN-Ringer's solution at $+60 \mathrm{mV}$ ( $\square$ ). For fluorescence labeling, $\tau=36.0 \pm 4.0 \mu \mathrm{m} \times \mathrm{min}$; for current decrease at $+60 \mathrm{mV}, \tau=32.1 \pm 3.9$ $\mu \mathrm{M} \times \min$. Fluorescence from uninjected oocytes was subtracted as background. Data are representative for $n=3$ experiments.
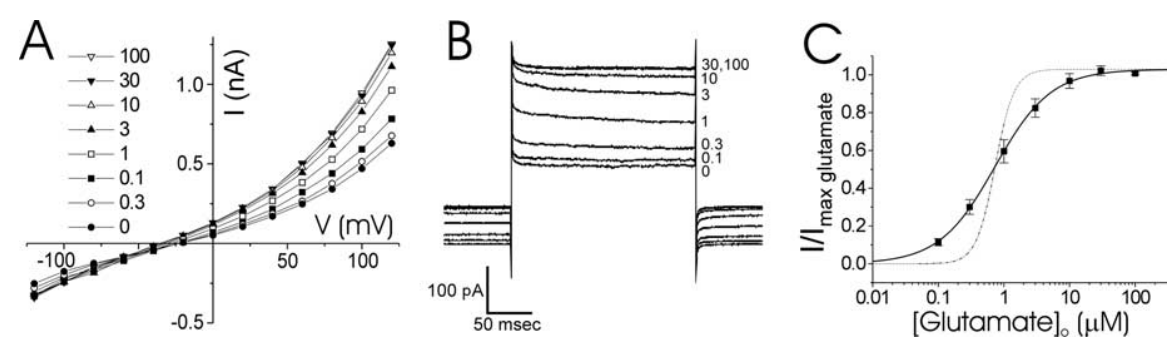

Figure 4. Glutamate-induced currents in HEK-293 cells expressing rat EAAT4. $\boldsymbol{A}$, Representative current-voltage relationships (

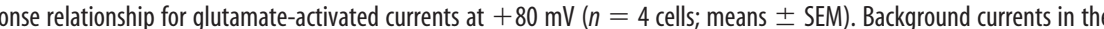
absence of glutamate were subtracted. Data were fit with the Hill equation (solid line) with $K_{\mathrm{m}}=0.73$ and $n_{\mathrm{H}}=1.02$. For comparison, dashed line represents fit with the Hill equation: $K_{\mathrm{m}}=0.73$ and $n_{\mathrm{H}}=3$.

\section{Discussion}

Based on the results presented above, binding of glutamate to each subunit in an EAAT trimer appears to independently gate the anion current; we found no evidence for intersubunit cooperativity in EAAT3 and EAAT4 for the activation of glutamate currents under physiological conditions. In contrast to a recent report (Torres-Salazar and Fahlke, 2006), we do not see sigmoidicity in the concentration dependence for glutamate activation of either the uptake currents or the anion currents. This is true even for anion currents recorded in thiocyanate, which introduced the most cooperativity in the above mentioned study (Torres-Salazar and Fahlke, 2006). Using FLIC, we demonstrate that EAAT subunits function independently in mediating glutamate uptake as well as glutamate-activated anion currents, at both low substrate concentrations and saturating substrate concentrations. Our data, however, do not rule out a common, centrally located anion channel in EAATs. Our findings only show that the anion channel(s) in an EAAT multimer are independently gated by the individual subunits. If the anion channel has a low maximum open probability $(\ll 100 \%)$ but each subunit can independently open the channel to its maximum conductance, then our data can still be explained by a centrally located anion pore shared by the three subunits. Indeed, the anion channel in EAAT1 was shown to have a very low maximum open probability (Wadiche and Kavanaugh, 1998). However, we favor the hypothesis that each subunit has an independent anion pore. In any case, our data show that, under physiological conditions, as well as in conditions in which $\mathrm{Na}^{+}$is the primary intracellular 
monovalent cation (such as may occur during ischemia), both the anion conductance and the glutamate uptake current must be gated independently by each individual subunit of the EAAT multimer.

The activation of EAAT subtypes with large anion conductances has been suggested to directly influence the excitability of presynaptic terminals in certain neurons (Picaud et al., 1995a; Palmer et al., 2003; Veruki et al., 2006; Wersinger et al., 2006). The effect that the EAAT-mediated anion current has on the excitability of the terminal depends not only on the glutamate affinity and on the relative conductance of the anion channel but also on the shape of the glutamate concentration dependence, i.e., whether the anion channel is activated cooperatively or independently by the individual subunits (Fig. $1 C, D$, insets). For a highly cooperative intersubunit interaction, the anion conductance will not be significantly activated at low glutamate concentrations. Conversely, in a mechanism of independent subunits, the anion conductance will be significantly more activated even at low glutamate concentrations (Fig. 1C,D, insets). Therefore, our finding that the anion conductance is independently activated by the different subunits leads to significantly different predictions of the effects that the EAAT-mediated anion currents can have on synaptic transmission versus if the anion conductance is cooperatively activated by the individual subunits in EAATs.

\section{References}

Arriza JL, Eliasof S, Kavanaugh MP, Amara SG (1997) Excitatory amino acid transporter 5, a retinal glutamate transporter coupled to a chloride conductance. Proc Natl Acad Sci USA 94:4155-4160.

Bergles DE, Tzingounis AV, Jahr CE (2002) Comparison of coupled and uncoupled currents during glutamate uptake by GLT-1 transporters. J Neurosci 22:10153-10162.

Brady JD, Rich ED, Martens JR, Karpen JW, Varnum MD, Brown RL (2006) Interplay between PIP3 and calmodulin regulation of olfactory cyclic nucleotide-gated channels. Proc Natl Acad Sci USA 103:15635-15640.

Danbolt NC (2001) Glutamate uptake. Prog Neurobiol 65:1-105.

Fairman WA, Vandenberg RJ, Arriza JL, Kavanaugh MP, Amara SG (1995) An excitatory amino-acid transporter with properties of a ligand-gated chloride channel. Nature 375:599-603.

Grewer C, Balani P, Weidenfeller C, Bartusel T, Tao Z, Rauen T (2005)
Individual subunits of the glutamate transporter EAAC1 homotrimer function independently of each other. Biochemistry 44:11913-11923.

Koch HP, Larsson HP (2005) Small-scale molecular motions accomplish glutamate uptake in human glutamate transporters. J Neurosci 25:1730-1736.

Larsson HP, Picaud SA, Werblin FS, Lecar H (1996) Noise analysis of the glutamate-activated current in photoreceptors. Biophys J 70:733-742.

Larsson HP, Tzingounis AV, Koch HP, Kavanaugh MP (2004) Fluorometric measurements of conformational changes in glutamate transporters. Proc Natl Acad Sci USA 101:3951-3956.

Mim C, Balani P, Rauen T, Grewer C (2005) The glutamate transporter subtypes EAAT4 and EAATs 1-3 transport glutamate with dramatically different kinetics and voltage dependence but share a common uptake mechanism. J Gen Physiol 126:571-589.

Palmer MJ, Taschenberger H, Hull C, Tremere L, von Gersdorff H (2003) Synaptic activation of presynaptic glutamate transporter currents in nerve terminals. J Neurosci 23:4831-4841.

Picaud S, Larsson HP, Wellis DP, Lecar H, Werblin F (1995a) Cone photoreceptors respond to their own glutamate release in the tiger salamander. Proc Natl Acad Sci USA 92:9417-9421.

Picaud SA, Larsson HP, Grant GB, Lecar H, Werblin FS (1995b) Glutamategated chloride channel with glutamate-transporter-like properties in cone photoreceptors of the tiger salamander. J Neurophysiol 74:1760-1771.

Torres-Salazar D, Fahlke C (2006) Intersubunit interactions in EAAT4 glutamate transporters. J Neurosci 26:7513-7522.

Veruki ML, Morkve SH, Hartveit E (2006) Activation of a presynaptic glutamate transporter regulates synaptic transmission through electrical signaling. Nat Neurosci 9:1388-1396.

Wadiche JI, Kavanaugh MP (1998) Macroscopic and microscopic properties of a cloned glutamate transporter/chloride channel. J Neurosci 18:7650-7661.

Wadiche JI, Arriza JL, Amara SG, Kavanaugh MP (1995a) Kinetics of a human glutamate transporter. Neuron 14:1019-1027.

Wadiche JI, Amara SG, Kavanaugh MP (1995b) Ion fluxes associated with excitatory amino acid transport. Neuron 15:721-728.

Wersinger E, Schwab Y, Sahel JA, Rendon A, Pow DV, Picaud S, Roux MJ (2006) The Glutamate transporter EAAT5 works as a presynaptic receptor in mouse rod bipolar cells. J Physiol (Lond) 577:221-234.

Yernool D, Boudker O, Jin Y, Gouaux E (2004) Structure of a glutamate transporter homologue from Pyrococcus horikoshii. Nature 431:811-818.

Zerangue N, Kavanaugh MP (1996) Flux coupling in a neuronal glutamate transporter. Nature 383:634-637. 